Wilhelm Kulisch

\title{
Deposition \\ of Diamond-Like \\ Superhard Materials
}

With 6o Figures 


\section{Contents}

1. The Material Property Hardness ............... 1

1.1 Definition of Hardness $\ldots \ldots \ldots \ldots \ldots \ldots \ldots \ldots \ldots \ldots \ldots$

1.2 Hardness Measurements ..................... 2



1.3.1 Ideal Materials ..................... 5

1.3 .2 Real Materials ..................... 8

1.4 Correlations with Other Material Properties ........... 12

1.4.1 Hardness versus Volumetric Lattice Energy . . . . . . 12

1.4.2 Hardness and Bulk Modulus ............... 13

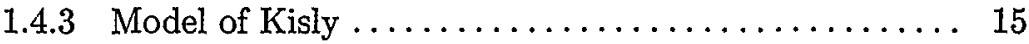

1.4.4 Model of Cohen ..................... 16

2. Diamond-Like Materials .................... 21

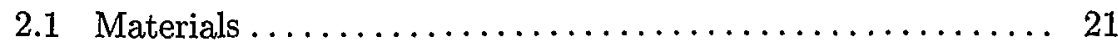

2.2 The $\mathrm{B} / \mathrm{C} / \mathrm{N}$ System $\ldots \ldots \ldots \ldots \ldots \ldots \ldots \ldots \ldots \ldots \ldots \ldots 21$

2.2.1 Materials in the $\mathrm{B} / \mathrm{C} / \mathrm{N}$ System $\ldots \ldots \ldots \ldots \ldots \ldots \ldots 22$

2.2 .2 Carbon and Boron Nitride ................. 23

2.3 Properties of Diamond-Like Materials . . . . . . . . . . 27

2.3.1 Thermal and Vibrational Properties ............ 29

2.3.2 Optical and Electronic Properties ............ 32

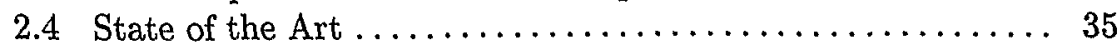

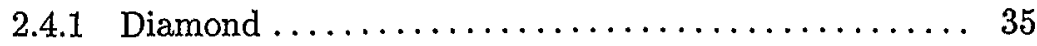

2.4.2 Tetrahedral Amorphous Carbon .............. 36



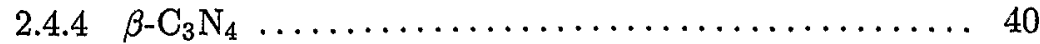

2.5 Organization of the Remainder of this Book $\ldots \ldots \ldots \ldots \ldots 41$

3. Growth Mechanisms ...................... 43

3.1 On the Deposition of Thin Films ................ 43

3.2 Growth of Diamond Films ................... 44

3.2.1 Methods for Low-Pressure Diamond Synthesis ...... 44





3.2 .4 Surface Processes...................... 63 
3.2.5 Macroscopic Diamond Growth ............ 74

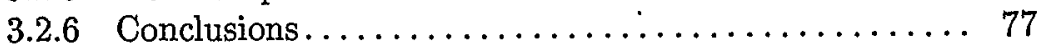

3.3 Growth of $\mathrm{c}-\mathrm{BN}$ Films $\ldots \ldots \ldots \ldots \ldots \ldots \ldots \ldots \ldots \ldots \ldots \ldots$

3.3.1 Methods and Parameters................ 79

3.3.2 Gas Phase and Transport Processes............ 87

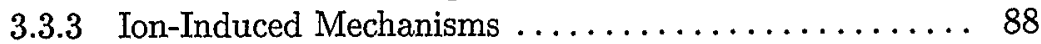

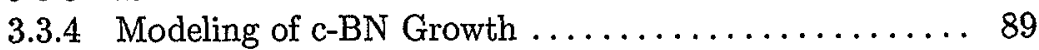

3.3.5 The Sputter Model ................... 93

3.3 .6 Conclusions......................... 101

3.4 Growth of ta-C Films . . . . . . . . . . . . . . . 105

3.4.1 Experimental Observations ............... 106

3.4 .2 Growth Mechanisms . . . . . . . . . . . . . 108

3.4 .3 Conclusions......................... 111

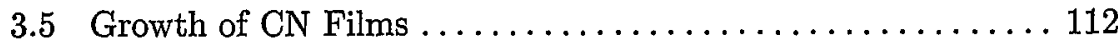

3.5.1 Experimental Observations ............... 112

3.5 .2 Discussion . . . . . . . . . . . . . . . . . . 117

3.6 Conclusions .......................... 120

4. Nucleation Mechanisms................... 125

4.1 On the Theory of Nucleation ................. 125

4.1.1 Macroscopic Thermodynamic Description ......... 125

4.1.2 Statistical Thermodynamic Description ......... 128

4.1.3 Atomistic Description .................. 129

4.1.4 Influence of Surface Defects . . . . . . . . . . . 131

4.1 .5 (Hetero)epitaxy .................... 132

4.1.6 Nucleation of Superhard Materials............. 133

4.2 Nucleation of Diamond . . . . . . . . . . . . . . 134

4.2 .1 General Considerations................... 134

4.2.2 Nucleation on Untreated Substrates . . . . . . . . 136

4.2.3 Nucleation on Highly-Oriented Graphite. . . . . . . . . 138

4.2.4 Abrasive Treatment ........................ 140

4.3 Bias-Enhanced Nucleation of Diamond . . . . . . . . . . 141

4.3.1 General Considerations..................... 141

4.3 .2 Experimental Observations .................. 144

4.3 .3 Discussion and Modeling ................. 155

4.4 Nucleation of c-BN . . . . . . . . . . . . . . . . . 159

4.4 .1 Experimental Observations ................. 160

4.4 .2 Mechanisms of Nucleation . . . . . . . . . . . . 163

4.5 Nucleation of ta-C and $\beta-\mathrm{C}_{3} \mathrm{~N}_{4} \ldots \ldots \ldots \ldots \ldots \ldots \ldots \ldots \ldots$

4.6 Conclusions........................... 168

References $\ldots \ldots \ldots \ldots \ldots \ldots \ldots \ldots \ldots \ldots \ldots \ldots \ldots \ldots \ldots \ldots \ldots$

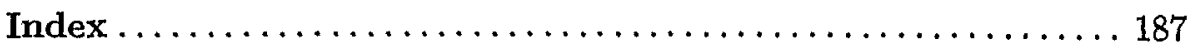

